\title{
Dynamical structure of fields in light cone coordinates
}

\author{
K. Kargar, ${ }^{1}$ A. Shirzad, ${ }^{2,3}$ and M. Monemzadeh ${ }^{1}$ \\ ${ }^{1}$ Department of Physics, University of Kashan, Kashan 87317-53153, Iran \\ ${ }^{2}$ Department of Physics, Isfahan University of Technology, Isfahan 87317-53153, Iran \\ ${ }^{3}$ School of Particles and Accelerators, Institute for Research in Fundamental Sciences (IPM), \\ Tehran 87317-53153, Iran
}

(Received 21 October 2018; published 26 February 2019)

\begin{abstract}
It is well known that additional constraints emerge in light cone coordinates. We enumerate the number of physical modes in light cone coordinates and compare it with conventional coordinates. We show that the number of Schrödinger modes is divided by two in light cone coordinates. We study the effect of this reduction in the number of ladder operators acting on physical states of a system. We analyze the scalar spinor, and vector field theories carefully to see the effect of changes in the dynamical structure of these theories from the view point of the reduction of Schrödinger modes in light-cone coordinates. In this way, we propose an alternative expansion of dynamical variables which defer from other literatures.
\end{abstract}

DOI: 10.1103/PhysRevD.99.045019

\section{INTRODUCTION}

Considering the various subgroups of the Poincaré group, in a pioneer paper in 1949 [1], Dirac introduced three forms for relativistic dynamics: instant form (I.F.), front form (F.F.), and point form (P.F.). These forms are related to the various choices of the time axis. The instant form is the usual choice of the coordinate $x^{0}$ as the time coordinate, while in the front form, $\left(x^{0}+x^{3}\right) / \sqrt{2}$ is chosen as the time coordinate. The front form has special features with so many applications in theoretical physics, especially in nonperturbative QCD [2], string theory [3], gravity [4], and so on. In the literature of high-energy physics, the front form is recognized by different names such as "light front," "infinite momentum frame," and "light cone." In this paper, we use "light cone." For a brief review of light-cone quantization and its application in high-energy physics, see Ref. [5].

In the light-cone formulation of physical systems, the hyperplane $x^{+}=\left(x^{0}+x^{3}\right) / \sqrt{2}$ acts as the equal time hyperplane. The light-cone coordinates are $x^{ \pm}=\left(x^{0} \pm x^{3}\right) /$ $\sqrt{2}=\left(x_{0} \mp x_{3}\right) / \sqrt{2}=x_{\mp}$ and $x^{\perp} \equiv\left(x^{1}, x^{2}\right)$. For an arbitrary four-vector $A^{\mu}$ with components $\left(A^{0}, \mathbf{A}\right)$, we define the light-cone components as $\left(A^{+}, A^{i}, A^{-}\right)=$ $\left(A^{+}, \tilde{A}\right)$, where $i=1,2$ and $A^{ \pm}=\left(A^{0} \pm A^{3}\right) / \sqrt{2}$ and $\tilde{A}=\left(A^{i}, A^{-}\right)$. So, for an invariant spacetime length element in Minkowski space, we have

Published by the American Physical Society under the terms of the Creative Commons Attribution 4.0 International license. Further distribution of this work must maintain attribution to the author(s) and the published article's title, journal citation, and DOI. Funded by SCOAP.

$$
d s^{2}=d x_{0}^{2}-d x_{3}^{2}-d x_{i}^{2}=2 d x_{+} d x_{-}-d x_{i}^{2}
$$

which shows that the metric has nondiagonal elements.

Historically, light-cone coordinates are well known for particle physicists since they are used to derive some QCD sum rules [6,7]. The large variety of applications of lightcone coordinates comes from the advantage of the relativistic dynamics of physical systems on the hyperplane of the $x_{0}+x_{3}=$ const. Dirac mentioned some of these advantages. First, in light-cone coordinates, there are seven kinematical Poincaré generators, while in the conventional formulation, only six are kinematical. Second, the nondiagonal form of the light-cone metric enables us to separate the total energy of a system of relativistic particles into center of mass energy and relative energy [5]. This is different from the instant form, in the sense that the appearance of the square root in the relation of energy, $P_{0}=\left(\vec{P}^{2}+M^{2}\right)^{1 / 2}$, prohibits a similar separation of variables. These advantages, and especially the latter one, have made the light-cone coordinates an appropriate tool for calculating quantities such as wave functions.

One special feature of using light-cone coordinates is the emergence of additional constraints compared to the conventional coordinates. We call these additional constraints light-cone constraints. This change in constraint structure of the theory is well known [2]. However, the number of light-cone constraints for a generic theory is not well understood yet. Physically, we expect no change in the dynamical content of the theory upon changing the coordinates of spacetime. So, one needs to identify clearly the role of light-cone coordinates in the dynamical behavior of the system. This is the main task of this work.

We will show explicitly that the light-cone constraints sit in place of half of the physical degrees of freedom (d.o.f.). 
Hence, the number of dynamical d.o.f. is divided by two, compared to the conventional coordinates. Although this phenomenon is met by physicists working on concrete models [5], it is not clearly recognized as a general rule for an arbitrary model. We will show that the light-cone constraints together with the remaining half of the dynamical equations of motion are equivalent to the whole equations of motion in conventional coordinates.

The next problem is how to choose the physical modes to be quantized in light-cone coordinates. For instance, some authors divide the momentum space into two parts and work with, say, the $k_{-}>0$ half of the momentum space [8]. This happens when one insists on expanding the fields with the same combination as in conventional coordinates. In this paper, we give another approach, in which we maintain the whole momentum space but put away half of the physical modes. In this approach, summation over spin in a spinor field and/or summation over polarization in a gauge field theory is no more necessary in light-cone coordinates. In other words, a light-cone observer is able to observe only one of the spin (polarization) states of an electron (photon).

In the remainder of this paper, we do the above task for the major type of physical theories which are quadratic or first order with respect to velocities. We show that, in both types of theories, the phenomenon of halving the number of dynamical modes is similar. In Sec. II, we find the general form of the constraint structure of a theory in light-cone coordinates and enumerate the number of dynamical variables. We do this both for second-order and first-order Lagrangians. Section III details the quantization procedure based on the symplectic approach of quantization, which is more or less a new approach in light-cone quantization. Sections IV and V deal with the same procedure for the special case of the spinor field theory and the vector field theory. In Sec. VI, we discuss the case of Yang-Mills theories and, at the end of this section, we try embedding the non-Abelian Yang-Mills theories in light-cone coordinates using the BFFT method. The last section provides our conclusions.

\section{NUMBER OF DYNAMICAL VARIABLES}

As we mentioned earlier, formulation of theories in lightcone coordinates leads to a different Hamiltonian structure in comparison with conventional coordinates [5]. Since in light-cone coordinates $x^{+}$is the time coordinate, the conjugate momentum is defined as

$$
\pi_{\mathrm{F} . \mathrm{F} .}=\frac{\partial \mathcal{L}}{\partial\left(\partial_{+} \phi\right)},
$$

which differs from the ordinary instant form momentum $\pi_{\mathrm{I} . \mathrm{F}}=\frac{\partial \mathcal{L}}{\partial\left(\partial_{0} \varphi\right)}$ in the sense that

$$
\pi_{\mathrm{FF}}=\frac{1}{\sqrt{2}}\left(\pi_{\mathrm{I} . \mathrm{F}}-\partial_{3} \varphi\right) .
$$

In addition to a different Hamiltonian structure, this point leads to a different number of dynamical variables. We investigate the problem in turns of two major important field theoretic systems, i.e., quadratic Lagrangians and firstorder Lagrangians (with respect to the velocities).

\section{A. Quadratic Lagrangian}

Consider a typical theory described by a set of dynamical fields $\phi_{a}(a=1,2, \ldots, n)$. Suppose the Lagrangian of the theory is at most quadratic with respect to the partial derivatives of the fields. Taking into account the Lorentz invariance, the most general form of the kinetic term is $g^{a b} \partial_{\mu} \phi_{a} \partial^{\mu} \phi_{b}$ for some symmetric matrix $g$. In conventional coordinates (instant form), we have $\mathcal{L}=g^{a b}\left(\partial_{0} \phi_{a} \partial_{0} \phi_{b}-\nabla \phi_{a} . \nabla \phi_{b}\right)+\cdots$, and the definition of momenta (i.e., $\pi_{\mathrm{I} . \mathrm{F}}^{a} \equiv 2 g^{a b} \partial_{0} \phi_{b}$ ) gives no constraint for nonsingular $g$. In the light-cone coordinates (front form), however, the kinetic term in the Lagrangian is written as $2 g^{a b}\left(\partial_{+} \phi_{a} \partial_{-} \phi_{b}-\partial_{\perp} \phi_{a} . \partial_{\perp} \phi_{b}\right)$, which gives the conjugate momentum $\pi_{\mathrm{F} . \mathrm{F}}^{a}=2 g^{a b} \partial_{-} \phi_{b}$. Since there is no velocity in this relation, we have the constraints

$$
\chi^{a} \equiv \pi_{\mathrm{I} . \mathrm{F}}^{a}-2 g^{a b} \partial_{-} \phi_{b} \simeq 0 .
$$

Hence, the nondiagonal form of the light-cone metric changes the constraint structure of the system. If the original theory is not constrained (e.g., Klein-Gordon theory), it will possess some new constraints, while a system which is already a constrained system in conventional coordinates (e.g., electromagnetism) will possess additional constraints due to the linearity of the Lagrangian with respect to the velocities $\partial_{+} \phi_{a}$.

Suppose there are $k$ first-class and $m$ second-class constraints on the phase space in conventional coordinates. We also need $k$ subsidiary conditions as gauge fixing conditions to reach the reduced phase space. Hence, there exist, all together, $2 k+m \equiv l$ conditions on the fields in phase space. The number of d.o.f. is, therefore, $2 n-l$ in Hamiltonian formalism and $n-l / 2$ in Lagrangian formalism [9].

Now, by going to the light-cone coordinates, the number of remaining d.o.f. in phase space should be divided by 2 . The reason is as follows: the $n-l / 2$ physical d.o.f. correspond to variables in the Lagrangian with truly quadratic terms with respect to the velocities in the conventional coordinates. As we showed, the quadratic terms with respect to conventional velocities (i.e., $\left.\left(\partial_{0} \phi_{a}\right)^{2}\right)$ are replaced by terms $\partial_{+} \phi_{a} \partial_{-} \phi_{a}$ in light-cone coordinates which are linear with respect to velocities. Hence, in the light-cone formulation of the theory, we will have $\frac{2 n-l}{2}$ additional constraints which we call "light-cone constraints."

The light-cone constraints are second class in the sense that their consistency with time determines the corresponding Lagrange multipliers. Since each second class constraint reduces one dynamical variable, we have 


$$
\mathcal{N}_{C}^{\mathrm{FF}}=l+\frac{2 n-l}{2}=n+\frac{l}{2},
$$

where $\mathcal{N}_{C}^{F}$.F is the total number of constraints in front form. In this way, half of the dynamical variables of the phase space are omitted by the light-cone constraints and the number of d.o.f. of the theory reduces to $\frac{2 n-l}{2}$. In subsequent sections, we will see this effect for Klein-Gordon and electromagnetic field theories.

However, note that we have not restricted the physical sector of the theory in phase space by going to the lightcone coordinates. To see this, we may try to project the additional constraints to the conventional phase space to see if there is any possible reduction. For this reason, we try to transform constraint $\pi_{\mathrm{F} . \mathrm{F}}-\partial_{-} \phi=0$ from light-cone coordinates to conventional coordinates. By using the chain rule for $\pi_{\mathrm{F} . \mathrm{F}}$, we obtain

$$
\begin{aligned}
\pi_{\mathrm{F} . \mathrm{F}} & =\frac{\partial \mathcal{L}}{\partial\left(\partial_{0} \phi\right)} \frac{\partial\left(\partial_{0} \phi\right)}{\partial\left(\partial_{+} \phi\right)}+\frac{\partial \mathcal{L}}{\partial\left(\partial_{3} \phi\right)} \frac{\partial\left(\partial_{3} \phi\right)}{\partial\left(\partial_{+} \phi\right)} \\
& =\frac{1}{\sqrt{2}}\left(\partial_{0} \phi-\partial_{3} \phi\right) .
\end{aligned}
$$

The right-hand side of this equation is the same as $\partial_{-} \phi$ and so we will get the trivial relation $0=0$. So any attempt to find an equivalent hyperplane for the constraint surface due to the light-cone constraints will lead to trivial equations in the conventional coordinates. In other words, there is no hyperplane in the conventional coordinate phase space equivalent to the hyperplane of light-cone constraints. Therefore the change in constraint structure in light-cone coordinates does not mean that the classical phase space in conventional coordinates is reduced.

\section{B. First-order Lagrangian}

The most well-known Lagrangian containing a Lorentz invariant first-order dynamical term includes $\bar{\psi}_{\alpha} \gamma_{\alpha \beta}^{\mu} \partial_{\mu} \psi_{\beta}(\alpha, \beta=1,2, \ldots, n)$ as appears in the familiar Dirac Lagrangian. To study the constraint structure of such field theories, we investigate the symplectic matrix of this theory in conventional coordinates as well as light-cone coordinates. By considering $\bar{\psi}_{\alpha}$ and $\psi_{\beta}$ as the independent variables of phase space, in conventional coordinates the dynamical term $\bar{\psi}_{\alpha} \gamma_{\alpha \beta}^{0} \partial_{0} \psi_{\beta}$ gives the symplectic matrix as (see Appendix A)

$$
\omega=\left(\begin{array}{cc}
0 & \gamma^{0}{ }_{n \times n} \\
-\gamma^{0}{ }_{n \times n} & 0
\end{array}\right) .
$$

Since $\operatorname{det}\left(\gamma^{0}\right) \neq 0$, so $\gamma^{0}$ does not have any null eigenvector. In conventional coordinates, the number of phase space d.o.f. is $2 n$. For example in ordinary Dirac fields, $n=4$ and the number of phase space variables is 8 .

In light-cone coordinates we set $\gamma^{\mu} \partial_{\mu}=\gamma^{+} \partial_{+}+\gamma^{-} \partial_{-}+$ $\gamma^{\perp} \partial_{\perp}$ where

$$
\gamma^{ \pm}=\frac{1}{\sqrt{2}}\left(\gamma^{0} \pm \gamma^{3}\right)
$$

The dynamical terms in the Lagrangian is $\bar{\psi}_{\alpha} \gamma_{\alpha \beta}^{+} \partial_{+} \psi_{\beta}$ which gives the symplectic matrix as

$$
\omega=\left(\begin{array}{cc}
0 & \gamma^{+}{ }_{n \times n} \\
-\gamma^{+}{ }_{n \times n} & 0
\end{array}\right) .
$$

In four-dimensional spacetime, all representations of Dirac matrices are unitarily equivalent, so it is sufficient to consider a specific representation and investigate the rank of $\gamma^{+}$. By choosing the chiral representation, we have [10],

$$
\gamma^{+}=\frac{1}{\sqrt{2}}\left(\begin{array}{cccc}
0 & 0 & 2 & 0 \\
0 & 0 & 0 & 0 \\
0 & 0 & 0 & 0 \\
0 & 2 & 0 & 0
\end{array}\right)
$$

which shows that $\gamma^{+}$has two null eigenvectors. Hence, in light-cone coordinates, the number of d.o.f. in the phase space is $n$ instead of $2 n$. In the case of the four-dimensional Dirac field, it is four instead of eight.

Thus, similar to the case of the second-order Lagrangian, the number of d.o.f. is divided by two in the light-cone formulation of field theories with the first-order Lagrangian. Therefore, the number of Schödinger modes in light-cone coordinates are half of those in the conventional coordinates.

\section{QUANTIZATION PROCEDURE}

Let us see the effect of change in the constraint structure on the classical dynamics, as well as the quantization procedure of the system. Classically, we want to know what happens to half of the d.o.f. which are absent in the lightcone coordinates. In conventional coordinates, we need to solve $2 n-l$ first-order differential equations for the dynamical variables in phase space. However, in lightcone coordinates, we have $n-l / 2$ constraints together with $n-l / 2$ first-order differential equations with respect to time. Hence, the total number of equations at hand is the same in both formalisms, and the physical results are the same, as it should be.

In fact, just the superficial features of the dynamical equations are different in two approaches; i.e., in conventional coordinates, all $2 n-l$ equations of motion include derivatives with respect to $x^{0}$, while in light-cone coordinates, $n-l / 2$ constraints do not include derivatives with respect to $x^{+}$and the remaining $n-l / 2$ do include derivatives with respect to $x^{+}$.

Our next goal is to find a suitable basis for the variables of the reduced phase space in order to follow the dynamics of the system. Suppose we are able to find a suitable basis for the whole phase space of the system, in which imposing 
the constraints leads to omitting a number of redundant variables. Such a basis is recognized in the literature of constrained systems as the Darbeaux basis [9]. Hence, in comparison with conventional coordinates, the additional light-cone constraints lead to a smaller reduced phase space with $n-l / 2$ dynamical ( $x^{+}$-dependent) modes. Then, we should solve the equations of motion to find the time dependence of physical modes in terms of $n-l / 2$ independent Schrödinger modes (see Appendix A).

In fact, in a Darbeaux basis, in light-cone coordinates, the procedure of solving the dynamics of the system will break into two steps: first, imposing the light-cone second class constraints to omit half of the d.o.f., and, second, solving the remaining equations of motion to find the Schrödinger modes. Finally, one needs to write the expansion of the fields in terms of the Schrödinger modes.

Since the Schrödinger modes play the role of creation and annihilation operators in the quantum theory, one may wonder if different number of Schrödinger modes in lightcone coordinates lead to a different quantum space of physical states. We expect the physical quantities should not depend on the choice of coordinate basis.

To answer this question, we should consider the commutation relations of ladder operators with the Hamiltonian of the system and compare the results in light-cone and conventional coordinates. As a familiar example, we quantize the Klein-Gordon theory in light-cone coordinates using the symplectic method. As we will see, in the lightcone coordinates, according to Eq. (5), we expect one constraint on the classical phase space of the theory which affects the quantization procedure.

The Klein-Gordon theory is introduced by the Lagrangian density

$\mathcal{L}=\frac{1}{2}\left(\partial_{\mu} \varphi \partial^{\mu} \varphi-m^{2} \varphi^{2}\right)=\partial_{+} \varphi \partial_{-} \varphi-\frac{1}{2}\left(\partial_{\perp} \varphi\right)^{2}-\frac{1}{2} m^{2} \varphi^{2}$.

The conjugate light-cone momentum is

$$
\pi \equiv \frac{\partial \mathcal{L}}{\partial\left(\partial_{+} \varphi\right)}=\partial_{-} \varphi
$$

which introduces the primary constraint $\chi \equiv \pi-\partial_{-} \varphi \simeq 0$ on the phase space. The total Hamiltonian [11] reads

$$
H_{T}=\int d^{3} \tilde{x}\left(\frac{1}{2}\left(\partial_{\perp} \varphi\right)^{2}+\frac{1}{2} m^{2} \varphi^{2}+u(x) \chi(x)\right),
$$

where $u(x)$ is the Lagrange multiplier. Assume the equal time fundamental Poisson brackets as

$$
\begin{aligned}
& \left\{\varphi(\tilde{x}), \varphi\left(\tilde{x}^{\prime}\right)\right\}_{x^{+}}=\left\{\pi(\tilde{x}), \pi\left(\tilde{x}^{\prime}\right)\right\}_{x^{+}}=0, \\
& \left\{\varphi(\tilde{x}), \pi\left(\tilde{x}^{\prime}\right)\right\}_{x^{+}}=\delta\left(x^{-}-x^{\prime-}\right) \delta^{2}\left(x^{\perp}-x^{\prime \perp}\right) .
\end{aligned}
$$

Since the constraint $\chi$ considered at different points constitutes a system of second class constraints, the consistency condition $\partial_{+} \chi(x)=\left\{\chi(x), H_{T}\right\}_{x^{+}}=0$ will not give a secondary constraint; instead, it determines the Lagrange multiplier via the equation

$$
2 \partial_{-} u(x)=\left(\partial_{\perp} \partial_{\perp} \varphi-m^{2} \varphi\right) .
$$

To impose the single constraint $\chi(x)$ on the fields, it is more suitable to use the following Fourier expansions,

$$
\begin{aligned}
\varphi & =\frac{1}{(2 \pi)^{3 / 2}} \int d^{3} \tilde{k} a\left(\tilde{k}, x^{+}\right) e^{i \tilde{k} \cdot \tilde{x}}, \\
\pi & =\frac{1}{(2 \pi)^{3 / 2}} \int d^{3} \tilde{k} c\left(\tilde{k}, x^{+}\right) e^{-i \tilde{k} \cdot \tilde{x}} .
\end{aligned}
$$

The physical modes are $a\left(\tilde{k}, x^{+}\right)$and $c\left(\tilde{k}, x^{+}\right)$. Imposing the light-cone constraint $\pi-\partial_{-} \varphi=0$ on the expansions (16) gives

$$
c\left(\tilde{k}, x^{+}\right)=-i k_{-} a\left(-\tilde{k}, x^{+}\right) .
$$

In contrast with conventional coordinates where there are two physical modes, in light-cone coordinates we have only one independent physical mode which we assume to be $a\left(\tilde{k}, x^{+}\right)$. Equation (17) shows that $c(\tilde{k})$ is determined in terms of $a(\tilde{k})$. Now using Eq. (A2), in Appendix A, to a construct symplectic two-form, we have

$$
\Omega=\int d^{3} \tilde{k}\left(-2 i k_{-}\right) d a\left(-\tilde{k}, x^{+}\right) \wedge d a\left(\tilde{k}, x^{+}\right) .
$$

Hence, the Dirac brackets of the physical modes are

$$
\left\{a\left(\tilde{k}, x^{+}\right), a\left(\tilde{k}^{\prime}, x^{+}\right)\right\}_{\text {D.B }}=\frac{-1}{2 i k_{-}} \delta^{3}\left(\tilde{k}+\tilde{k}^{\prime}\right) .
$$

In terms of the physical modes $a\left(\tilde{k}, x^{+}\right)$, the canonical Hamiltonian is

$$
H_{c}=\int d^{3} \tilde{k} \frac{1}{2}\left(k_{\perp}^{2}+m^{2}\right) a\left(\tilde{k}, x^{+}\right) a\left(-\tilde{k}, x^{+}\right) .
$$

Using the canonical Hamiltonian, we are able to write equations of motion of the physical modes as

$$
\dot{a}\left(\tilde{k}, x^{+}\right)=\{a, H\}=i \omega_{+} a\left(\tilde{k}, x^{+}\right),
$$

where

$$
\omega_{+} \equiv \frac{k_{\perp}^{2}+m^{2}}{2 k_{-}}
$$

The solution of Eq. (21) is 


$$
a\left(\tilde{k}, x^{+}\right)=a(\tilde{k}, 0) e^{i \omega_{+} x^{+}}
$$

In contrast with the conventional coordinates where we deal with two coupled first-order differential equations of motion, in light-cone coordinates, we have only one differential equation. As we mentioned earlier, imposing the light-cone constraint (12), is equivalent to solving one equation of motion of ordinary coordinates. The original fields can be expanded in terms of the Schrödinger modes $a(\tilde{k}, 0)$ as

$$
\begin{aligned}
& \varphi\left(\tilde{x}, x^{+}\right)=\frac{1}{(2 \pi)^{3 / 2}} \int d^{3} \tilde{k} a(\tilde{k}, 0) e^{i k x}, \\
& \pi\left(\tilde{x}, x^{+}\right)=\frac{1}{(2 \pi)^{3 / 2}} \int d^{3} \tilde{k}\left(-i k_{-}\right) a(-\tilde{k}, 0) e^{-i k x} .
\end{aligned}
$$

By using Eq. (19) and the Dirac quantization prescription $\{,\} \rightarrow-i[$,$] , the quantum commutators of the Schrödinger$ modes as well as original fields can be written as

$$
\begin{gathered}
{\left[a(\tilde{k}), a\left(\tilde{k}^{\prime}\right)\right]=\frac{-1}{2 k_{-}} \delta^{3}\left(\tilde{k}+\tilde{k}^{\prime}\right),} \\
{\left[\varphi\left(\tilde{x}, x^{+}\right), \pi\left(\tilde{y}, x^{+}\right)\right]=\frac{1}{2} \delta^{3}(\tilde{x}-\tilde{y}),} \\
{\left[\varphi\left(\tilde{x}, x^{+}\right), \varphi\left(\tilde{y}, x^{+}\right)\right]=\frac{1}{2} \theta\left(x^{-}-y^{-}\right) \delta^{2}\left(x^{\perp}-y^{\perp}\right) .}
\end{gathered}
$$

where $\theta\left(x^{-}-y^{-}\right)$is the Heaviside step function.

In contrast to conventional coordinates, Eq. (27) shows that the field $\phi$ does not commute with itself on an equal light-cone time hyperplane. Let us investigate this property carefully. We want to find the commutation relation (27) from the nonequal time commutation relation of KleinGordon fields in conventional coordinates. In conventional coordinates, we have [10]

$[\varphi(x), \varphi(y)]_{\mathrm{I} . \mathrm{F}}=\int d^{3} \mathbf{k} \frac{1}{2 \omega_{\mathbf{k}}}\left(-e^{-i k(x-y)}+e^{i k(x-y)}\right)$,

which can be written covariantly as

$$
\begin{aligned}
& {\left[\varphi(x), \varphi(y]_{\mathrm{I} . \mathrm{F}}\right.} \\
& \quad=\int d^{4} k \delta\left(k^{2}-m^{2}\right) \theta\left(k_{0}\right)\left(-e^{-i k(x-y)}+e^{i k(x-y)}\right) .
\end{aligned}
$$

Note that the subscription I.F in Eq. (29) is no more necessary in covariant form of the commutation relations. Hence, we can transform this integral to a light-cone coordinate. By transforming $\delta\left(k^{2}-m^{2}\right)$ to light-cone coordinates and integrating over $k_{+}$, we have

$$
\begin{aligned}
{[\varphi(x), \varphi(y)]=} & \int d k_{-} d^{2} k_{\perp} \frac{1}{2 k_{-}}\left(-e^{-i k(x-y)}+e^{i k(x-y)}\right) \\
& \times\left.\theta\left(\frac{k_{+}+k_{-}}{\sqrt{2}}\right)\right|_{k_{+}=\frac{k_{\perp}^{2}+m^{2}}{2 k_{-}}} .
\end{aligned}
$$

Putting $x^{+}=y^{+}$, we can find the equal light-cone time commutation relations as

$$
\left[\varphi\left(\tilde{x}, x^{+}\right), \varphi\left(\tilde{y}, x^{+}\right)\right]=\frac{1}{2} \theta\left(x^{-}-y^{-}\right) \delta^{2}\left(x^{\perp}-y^{\perp}\right),
$$

which is exactly the commutation relation (27) we obtained by direct calculation in the light-cone coordinates. This simple result which shows the consistency of formulation of the Klein-Gordon theory in light-cone and conventional coordinate systems, although expected intuitively, is not shown explicitly in the literature yet. Note that the transformation from light-cone to conventional coordinates is not an ordinary Lorentz transformation.

Now we will turn back to the problem of interpreting a different number of ladder operators in light-cone and conventional coordinates. Let us see how we can interpret a different number of Schrödinger modes in light-cone coordinates.

Consider the commutation relation of ladder operators with the Hamiltonian in both coordinates. In conventional coordinates, we have

$$
\begin{gathered}
{\left[A(\mathbf{k}), A^{\dagger}(\mathbf{k})\right]=(2 \pi)^{3} \delta^{3}\left(\mathbf{k}-\mathbf{k}^{\prime}\right),} \\
{[H, A(\mathbf{k})]=-\omega_{\mathbf{k}} A(\mathbf{k}),} \\
{\left[H, A^{\dagger}(\mathbf{k})\right]=\omega_{\mathbf{k}} A^{\dagger}(\mathbf{k}) .}
\end{gathered}
$$

where $\omega_{\mathbf{k}}$ is the time component of the momentum 4-vector. As we see, the sign of the right-hand sides of Eqs. (32) and (33) are different for the annihilation and creation operators. In conventional coordinates, the onshell condition reads $\omega_{\mathbf{k}}^{2}=k_{0}^{2}=\mathbf{k}^{2}+m^{2}$; hence, the sign of the spacial components of momentum do not determine the sign of $\omega_{\mathbf{k}}$.

In the light-cone coordinates, however, the number of the ladder operators is divided by 2 and the commutation relations are

$$
\begin{aligned}
{\left[a(\tilde{k}), a\left(\tilde{k}^{\prime}\right)\right] } & =\frac{1}{2 k_{-}} \delta^{3}\left(\tilde{k}+\tilde{k}^{\prime}\right), \\
{[H, a(\tilde{k})] } & =-\omega_{\tilde{k}} a(\tilde{k}) .
\end{aligned}
$$

Remembering Eq. (22) shows that the sign of $\omega_{\tilde{k}}$ depends on the sign of $k_{-}$. This property divides the momentum space into two parts, $k_{-}>0$ and $k_{-}<0$, where $a(\tilde{k})$ is a creation operator in the $k_{-}>0$ region and an annihilation operator in the $k_{-}<0$ region. This point of view differs 
from the conventional approach [12-14] which insists on introducing two sets of ladder operators for creation and annihilation on the price of restricting the physical domain of momentum coordinate $k_{-}$to the region $k_{-}>0$.

Let us investigate the effect of light-cone ladder operators on the total momentum of the system. Using the definition of the energy-momentum tensor, $T_{\nu}^{\mu}=\frac{\partial \mathcal{L}}{\partial\left(\partial_{\mu} \varphi\right)} \partial_{\nu} \varphi-\mathcal{L} \delta_{\nu}^{\mu}$, the components of momentum in the light-cone coordinates are

$$
\begin{aligned}
P^{+} & =\int d^{3} \tilde{x} \pi \partial_{-} \varphi=\int d^{3} \tilde{k}\left(k_{-}^{2}\right) a(-k) a(k), \\
P^{i} & =\int d^{3} \tilde{x}\left(-\pi \partial_{-} \varphi\right)=-\int d^{3} \tilde{k} k_{-} k_{i} a(-k) a(k), \quad i=1,2 .
\end{aligned}
$$

Using commutation relation (25), we have

$$
\begin{aligned}
& {\left[P^{+}, a(\tilde{k})\right]=\left[P_{-}, a(\tilde{k})\right]=k_{-} a(\tilde{k}) .} \\
& {\left[P_{i}, a(\tilde{k})\right]=-\left[P^{i}, a(\tilde{k})\right]=k_{i} a(\tilde{k}) .}
\end{aligned}
$$

These relations verify the interpretation of $a(\tilde{k})$ with $k_{-}>0\left(k_{-}<0\right)$ as creation (annihilation) operators.

\section{SYMPLECTIC LIGHT-CONE QUANTIZATION OF SPINOR FIELDS}

Dirac theory is introduced by the first-order Lagrangian density

$$
\mathcal{L}=\bar{\psi}\left(i \gamma^{\mu} \partial_{\mu}-m\right) \psi .
$$

To quantize this theory in light-cone coordinates, it is convenient to use a decomposition of spinor space by the projection operators [8],

$$
\Lambda^{ \pm}=\frac{1}{2} \gamma^{\mp} \gamma^{ \pm}=\frac{1}{\sqrt{2}} \gamma^{0} \gamma^{ \pm}
$$

which project the spinor field $\psi$ to $\psi_{ \pm}=\Lambda^{ \pm} \psi$. Using the identities

$$
\gamma^{0} \gamma^{+}=\gamma^{-} \gamma^{0}, \quad \Lambda^{ \pm} \Lambda^{\mp}=0,
$$

we have $\gamma^{0} \psi_{+}=\frac{\sqrt{2}}{2} \gamma^{+} \psi_{+}$. Hence, the Lagrangian density of the Dirac field decomposes as

$$
\begin{aligned}
\mathcal{L}= & i \sqrt{2} \psi_{+}^{\dagger} \partial_{+} \psi_{+}+i \sqrt{2} \psi_{-}^{\dagger} \partial_{-} \psi_{-} \\
& -\psi_{-}^{\dagger}\left(m+i \gamma^{i} \partial_{i}\right) \gamma^{0} \psi_{+}-\psi_{+}^{\dagger}\left(m+i \gamma^{i} \partial_{i}\right) \gamma^{0} \psi_{-} .
\end{aligned}
$$

In this way, the Lagrangian (38) can be written as

$$
\mathcal{L}=i \sqrt{2} \psi_{+}^{\dagger} \partial_{+} \psi_{+}-\psi_{-}^{\dagger} \chi_{1}-\mathcal{H}_{c},
$$

where the density of the canonical Hamiltonian is

$$
\mathcal{H}_{c}=\frac{1}{\sqrt{2}} \psi_{+}^{\dagger}\left(m+i \gamma^{i} \partial_{i}\right) \gamma^{-} \psi_{-}
$$

In the above Lagrangian, the only dynamical variables are $\psi_{+}$and $\psi_{+}^{\dagger}$, while the equations of motion for the variables $\psi_{-}^{\dagger}$ and $\psi_{-}$give the constraints

$$
\begin{aligned}
& \chi_{1} \equiv i \partial_{-} \psi_{-}-\frac{1}{2}\left(m+i \gamma^{i} \partial_{i}\right) \gamma^{+} \psi_{+} \approx 0, \\
& \chi_{2} \equiv i \partial_{-} \psi_{-}^{\dagger}+\frac{1}{2}\left(m \psi_{+}^{\dagger}-i \partial_{i} \psi_{+}^{\dagger} \gamma^{i}\right) \gamma^{-} \approx 0 .
\end{aligned}
$$

In order to write a suitable mode expansion of the fields $\psi_{+}$ and $\psi_{+}^{\dagger}$, we look for a complete set of eigenfunctions of the Hamiltonian of the first quantized theory. In conventional coordinates, $u(\mathbf{k}) e^{\mathbf{i} \mathbf{k} . \mathbf{x}}$ and $v(\mathbf{k}) e^{-\mathbf{i k} . \mathbf{x}}$ are the eigenfunctions of Dirac Hamiltonian $h_{D}$,

$$
h_{D}=-i \gamma^{0} \gamma^{i} \partial_{i}+m \gamma^{0} \quad i=1,2,3,
$$

with the energy eigenvalues $E_{k}$ and $-E_{k}$, respectively [10]. Actually, the solutions of the eigenvalue equations $h_{D} \psi(x)= \pm E_{k} \psi(x)$ can be considered as $u(k) e^{-i k \cdot x}$ and $v(k) e^{i k . x}$ such as

$$
\begin{aligned}
& \left(\gamma^{\mu} k_{\mu}-m\right) u(k)=0, \\
& \left(\gamma^{\mu} k_{\mu}+m\right) v(k)=0 .
\end{aligned}
$$

Each of the equations (46) and (47) have two independent solutions distinguished by the eigenvalues of the component of spin operator, say in the third direction, i.e., $\Sigma^{3}$. Hence, for every solution of (46) and (47), we can decompose $u^{1}$ and $u^{2}$ as well as $v^{1}$ and $v^{2}$ by using the projection operators

$$
\mathcal{S}^{\mp}=\frac{1}{2}\left(1 \pm \Sigma^{3}\right) .
$$

In this way for the Dirac fields $\psi(x)$ and $\bar{\psi}(x)$ with eight independent phase space variables, we can set the eight eigenspinors $\left\{u_{s}, v_{s}, u_{s}^{\dagger}, v_{s}^{\dagger}\right\}$ for $s=1,2$. So, the summation over spin indices is necessary in the conventional coordinates.

On the other hand, in the light-cone coordinates, due to additional constraints (44), the dimension of the reduced phase space is four. So, in order to expand independent phase space variables in term of energy eigenfunctions, we need four energy eigenfunctions of the Hamiltonian operator. To do this, notice that the Dirac light-cone Hamiltonian operator can be recognized from the canonical Hamiltonian (43) as 


$$
h_{D}^{\mathrm{L} . \mathrm{C}} \equiv \frac{1}{\sqrt{2}}\left(m+i \gamma^{i} \partial_{i}\right) \gamma^{-}
$$

Using the plane wave solutions (46) and (47) we can introduce $u_{ \pm}(k)=\Lambda^{ \pm} u(k)$ and $v_{ \pm}(k)=\Lambda^{ \pm} v(k)$. Then it is easy to see

$$
\begin{aligned}
& h_{D}^{\mathrm{L} . \mathrm{C}} u_{+}(k)=k_{+} u_{+}(k), \\
& h_{D}^{\mathrm{L} .} \cdot u_{-}(k)=0, \\
& h_{D}^{\mathrm{L} . \mathrm{C}} v_{+}(k)=-k_{+} v_{+}(k), \\
& h_{D}^{\mathrm{L} \cdot \mathrm{C}} v_{-}(k)=0 .
\end{aligned}
$$

As is seen, spinors $u_{-}(k)$ and $v_{-}(k)$ are ruled out from the eigenspinors of $h_{D}^{\mathrm{L} . \mathrm{C}}$. On the other hand, spinors $\left\{u_{+}, v_{+}, u_{+}^{\dagger}, v_{+}^{\dagger}\right\}$ form a basis for the four-dimensional space of variables $\psi_{+}$and $\psi_{+}^{\dagger}$. In other words, in light-cone coordinates in contrast with conventional coordinates, there is the natural projection operator $\Lambda^{ \pm}$for the energy eigenspinors.

In this way there is no need to use the spin projection operators (48) to distinguish the degenerate spinors. Hence, there is no spin summation in expansion of the Dirac fields. Now we are able to expand the dynamical fields $\psi_{+}$and $\psi_{+}^{\dagger}$ in the basis $u_{+}(k) e^{-i \tilde{k} . \tilde{x}}$ and $v_{+}(k) e^{i \tilde{k} . \tilde{x}}$ and their conjugates as follows:

$$
\begin{aligned}
& \psi_{+}\left(\tilde{x}, x^{+}\right) \\
& \quad=\int d^{3} \tilde{k}\left(A\left(\tilde{k}, x^{+}\right) u_{+}(k) e^{-i \tilde{k} \cdot \tilde{x}}+B^{\dagger}\left(\tilde{k}, x^{+}\right) v_{+}(k) e^{i \tilde{k} . \tilde{x}}\right),
\end{aligned}
$$

$$
\begin{aligned}
& \psi_{+}^{\dagger}\left(\tilde{x}, x^{+}\right) \\
& \quad=\int d^{3} \tilde{k}\left(A^{\dagger}\left(\tilde{k}, x^{+}\right) u_{+}^{\dagger}(k) e^{i \tilde{k} . \tilde{x}}+B\left(\tilde{k}, x^{+}\right) v_{+}^{\dagger}(k) e^{-i \tilde{k} . \tilde{x}}\right) .
\end{aligned}
$$

Before going through the expansion of the fields, let us see what has happened to the state of the eigenstates with spinors $u_{+}(k)$ and $v_{+}(k)$. For this reason, consider the spin states of energy eigenfunctions in the rest frame $k_{r}=\frac{1}{\sqrt{2}}(m, 0,0, m)$. By choosing the rest frame in the relations (46) and (47), we simply have the solutions

$$
u_{+}=\left(\begin{array}{c}
1 \\
0 \\
0 \\
1
\end{array}\right), \quad v_{+}=\left(\begin{array}{c}
1 \\
0 \\
0 \\
-1
\end{array}\right)
$$

Compare these with the conventional basis $u^{1}$ and $u^{2}$ as $v^{1}$ and $v^{2}$ in the rest frame as $u^{1}=\left(\begin{array}{l}1 \\ 0 \\ 1 \\ 0\end{array}\right), \quad u^{2}=\left(\begin{array}{l}0 \\ 1 \\ 0 \\ 1\end{array}\right), \quad v^{1}=\left(\begin{array}{c}1 \\ 0 \\ -1 \\ 0\end{array}\right), \quad v^{2}=\left(\begin{array}{c}0 \\ 1 \\ 0 \\ -1\end{array}\right)$,

where $\quad \Sigma^{3} u^{1}=u^{1}, \quad \Sigma^{3} u^{2}=-u^{2}, \quad \Sigma^{3} v^{1}=v^{1}, \quad$ and $\Sigma^{3} v^{2}=-v^{2}$. It is easy to see $\Lambda^{+} u^{1}=\Lambda^{+} v^{1}$ and $\Lambda^{+} u^{2}=-\Lambda^{+} v^{2}$. This says that the spin states for positive and negative frequency solutions of conventional coordinates are no longer independent after projecting with the operator $\Lambda^{+}$. Hence, we can recognize the combination of spin states as

$$
\begin{aligned}
& u_{+}=\Lambda^{+}\left(u^{1}+u^{2}\right)=\Lambda^{+}\left(v^{1}-v^{2}\right), \\
& v_{+}=\Lambda^{+}\left(v^{1}+v^{2}\right)=\Lambda^{+}\left(u^{1}-u^{2}\right) .
\end{aligned}
$$

So, energy eigenfunctions $u_{+}$and $v_{+}$are projections of some combinations of spin states. Therefore, Schrödinger modes (or equivalently ladder operators in quantum theory) create and annihilate particles and antiparticles in specific superposition of spin states. This property is in contrast to the quantized Dirac fields in conventional coordinates.

For the dependent fields $\psi_{-}$and $\psi_{-}^{\dagger}$, using the constraints (44), we simply have

$$
\begin{aligned}
& \psi_{-}\left(\tilde{x}, x^{+}\right) \\
& =\int d^{3} \tilde{k}\left(A\left(\tilde{k}, x^{+}\right) u_{-}(k) e^{-i \tilde{k} \cdot \tilde{x}}+B^{\dagger}\left(\tilde{k}, x^{+}\right) v_{-}(k) e^{i \tilde{k} \cdot \tilde{x}},\right) \\
& \psi_{-}^{\dagger}\left(\tilde{x}, x^{+}\right) \\
& =\int d^{3} \tilde{k}\left(A^{\dagger}\left(\tilde{k} \cdot \tilde{x}, x^{+}\right) u_{-}^{\dagger}(k) e^{i \tilde{k} \cdot \tilde{x}}+B\left(\tilde{k}, x^{+}\right) v_{-}^{\dagger}(k) e^{-i \tilde{k} \cdot \tilde{x}}\right),
\end{aligned}
$$

where

$$
\begin{aligned}
& u_{-}(k)=\frac{m+\gamma^{i} k_{i}}{2 k_{-}} \gamma^{+} u_{+}(k), \\
& v_{-}(k)=\frac{m-\gamma^{i} k_{i}}{2 k_{-}} \gamma^{+} v_{+}(k) .
\end{aligned}
$$

These relations are also consistent with the relations (46) and (47). To find out the odd Poisson brackets of physical modes, we construct the symplectic two-form $\Omega=$ $\int d^{3} x i \sqrt{2} d \psi_{+}^{\dagger} \wedge d \psi_{+}$by using Eqs. (4) and (52) as follows: 


$$
\begin{aligned}
\Omega= & \int d^{3} \tilde{k} i \sqrt{2}\left(d A^{\dagger}\left(\tilde{k}, x^{+}\right) \wedge d A\left(\tilde{k}, x^{+}\right)\right. \\
& \left.-d B^{\dagger}\left(\tilde{k}, x^{+}\right) \wedge d B\left(\tilde{k}, x^{+}\right)\right) .
\end{aligned}
$$

So the odd Poisson brackets of physical modes read

$$
\begin{aligned}
& \left\{A\left(\tilde{k}, x^{+}\right), A^{\dagger}\left(\tilde{k}^{\prime}, x^{+}\right)\right\}_{+}=\frac{-i}{\sqrt{2}} \delta^{3}\left(\tilde{k}-\tilde{k}^{\prime}\right), \\
& \left\{B\left(\tilde{k}, x^{+}\right), B^{\dagger}\left(\tilde{k}^{\prime}, x^{+}\right)\right\}_{+}=\frac{i}{\sqrt{2}} \delta^{3}\left(\tilde{k}-\tilde{k}^{\prime}\right) .
\end{aligned}
$$

The canonical Hamiltonian (43) in terms of physical modes can be written as

$$
\begin{aligned}
\mathcal{H}_{c}= & \int d^{3} \tilde{k} \frac{m^{2}+k_{i}^{2}}{2 k_{-}}\left(A^{\dagger}\left(\tilde{k}, x^{+}\right) A\left(\tilde{k}, x^{+}\right)\right. \\
& \left.-B^{\dagger}\left(\tilde{k}, x^{+}\right) B\left(\tilde{k}, x^{+}\right)\right) .
\end{aligned}
$$

Using the above Hamiltonian and the algebra (60), the equations of motion of physical modes become

$$
\begin{aligned}
\partial_{+} A\left(\tilde{k}, x^{+}\right) & =-i \omega_{+} A\left(\tilde{k}, x^{+}\right), \\
\partial_{+} A^{\dagger}\left(\tilde{k}, x^{+}\right) & =i \omega_{+} A^{\dagger}\left(\tilde{k}, x^{+}\right), \\
\partial_{+} B\left(\tilde{k}, x^{+}\right) & =-i \omega_{+} B\left(\tilde{k}, x^{+}\right), \\
\partial_{+} B^{\dagger}\left(\tilde{k}, x^{+}\right) & =i \omega_{+} B^{\dagger}\left(\tilde{k}, x^{+}\right),
\end{aligned}
$$

where $\omega_{+}=\frac{m^{2}+k_{i}^{2}}{2 k_{-}}$. By writing the solutions of Eq. (62) in terms of Schrödinger modes and inserting them into the Eqs. (4) and (52), we have

$$
\begin{aligned}
& \psi_{+}\left(\tilde{x}, x^{+}\right)=\int d^{3} \tilde{k}\left(A(\tilde{k}) u_{+}(k) e^{-i k x}+B^{\dagger}(\tilde{k}) v_{+}(k) e^{i k x}\right), \\
& \psi_{+}^{\dagger}\left(\tilde{x}, x^{+}\right)=\int d^{3} \tilde{k}\left(A^{\dagger}(\tilde{k}) u_{+}^{\dagger}(k) e^{i k x}+B(\tilde{k}) v_{+}^{\dagger}(k) e^{-i k x}\right) .
\end{aligned}
$$

Similar results can be written for $\psi_{-}$and $\psi_{-}^{\dagger}$ where $u_{-}$and $v_{-}$are derived as in Eq. (58).

Using $\psi=\psi_{+}+\psi_{-}$and $u=u_{+}+u_{-}$, the expansions of original Dirac fields become

$$
\begin{aligned}
\psi\left(\tilde{x}, x^{+}\right) & =\int d^{3} \tilde{k}\left(A(\tilde{k}) u(k) e^{-i k x}+B^{\dagger}(\tilde{k}) v(k) e^{i k x}\right), \\
\psi^{\dagger}\left(\tilde{x}, x^{+}\right) & =\int d^{3} \tilde{k}\left(A^{\dagger}(\tilde{k}) u^{\dagger}(k) e^{i k x}+B(\tilde{k}) v^{\dagger}(\tilde{k}) e^{-i k x}\right) .
\end{aligned}
$$

As we mentioned earlier, in light-cone coordinates, the summation over spin states is no longer necessary in the expansions of the fields. This property is due to additional constraints (44) which appears in light-cone coordinates.
Also, the same situation arises in light-cone electromagnetic theory, where we need not choose any polarization vector to quantize this theory.

\section{SYMPLECTIC LIGHT-CONE QUANTIZATION OF VECTOR FIELDS}

The familiar electromagnetic theory is a gauge theory with two first class constraints in the conventional coordinates. Let us investigate the constraint structure of this theory in light-cone coordinates.

The Lagrangian $-\frac{1}{4} F^{\mu \nu} F_{\mu \nu}$ of electromagnetic theory should be written in light-cone coordinates as

$$
\mathcal{L}=\frac{1}{2} F_{+-} F_{+-}+F_{-i} F_{+i}-\frac{1}{4}\left(F_{i j}\right)^{2} .
$$

The conjugate momenta are

$$
\begin{gathered}
\pi^{+}=\frac{\partial \mathcal{L}}{\partial\left(\partial_{+} A_{+}\right)}=0, \\
\pi^{-}=\frac{\partial \mathcal{L}}{\partial\left(\partial_{+} A_{-}\right)}=F_{+-}, \\
\pi^{i}=\frac{\partial \mathcal{L}}{\partial\left(\partial_{+} A_{-}\right)}=F_{-i} \quad i=1,2,
\end{gathered}
$$

which give the primary constraints in the light-cone phase space as follows:

$$
\begin{gathered}
\chi_{0} \equiv \pi^{+} \simeq 0 \\
\chi_{i} \equiv \pi^{i}-F_{-i} \simeq 0 \quad i=1,2 .
\end{gathered}
$$

The total Hamiltonian reads

$$
\begin{aligned}
H_{T} & =\int d^{3} \tilde{x}\left(\frac{1}{2}\left(\pi^{-}\right)^{2}+\pi^{-} \partial_{-} A_{+}+\pi^{i} \partial_{i} A_{+}\right. \\
& \left.+\frac{1}{4} F_{i j} F_{i j}+u(x) \pi^{+}+v_{i}(x)\left(\pi^{i}-F_{-i}\right)\right),
\end{aligned}
$$

where $u(x)$ and $v_{i}(x)$ are Lagrange multipliers. Assuming the fundamental Poisson brackets as

$$
\left\{A_{\mu}\left(\tilde{x}, x^{+}\right), \pi^{\nu}\left(\tilde{y}, x^{+}\right)\right\}=\delta_{\mu}^{\nu} \delta^{3}(\tilde{x}-\tilde{y}),
$$

the consistency condition of the constraint $\chi_{0}$ gives the secondary constraint

$$
\phi_{0} \equiv \partial_{i} \pi^{i}+\partial_{-} \pi^{-} \approx 0,
$$

while the consistency of the constraints $\chi_{i}$ determines the Lagrange multipliers $v_{i}$ via 


$$
2 \partial_{-} v_{i}=\partial_{i} \pi^{-}-\partial_{j} F_{i j} .
$$

The consistency of the secondary constraint $\phi$ does not lead to a new constraint. Hence, we have two first class constraints $\chi_{0}$ and $\phi_{0}$ and two second class constraints $\chi_{i}$. Comparing with our general discussion on the number of d.o.f. in Sec. II, here we have $n=4$ physical fields $A^{\mu}$ with $k=2$ first class constraints $\pi_{0}$ and $\partial_{i} \pi_{i}(i=1,2,3)$, and no second class constraint in the conventional coordinates. The first class constraints $\chi_{0}$ and $\phi_{0}$ above are similar to the first class constraints in conventional coordinates. The number of phase space d.o.f. in conventional coordinates is $2 n-2 k=4$. However, the number of d.o.f. is divided by two in light-cone coordinates due to two additional constraints $\chi_{i}$ which does not have any counterpart in conventional coordinates.

To construct the reduced phase space of the system, we need two gauge fixing conditions conjugate to our two first class constraints. We begin with the gauge fixing condition $\omega_{1} \equiv A_{-} \approx 0$, which is, in fact, conjugate to the secondary constraint $\phi_{0}$. The consistency condition of this gauge, i.e., $\partial_{+} A_{-} \approx 0$, gives

$$
\omega_{2} \equiv \pi^{-}+\partial_{-} A_{+} \approx 0,
$$

which is the second required gauge fixing condition. Consistency of $\omega_{2}$ gives an equation to determine the Lagrange multiplier $u(x)$. By imposing the four constraints and two gauge fixing conditions, one obtains a reduced phase space with only two field variables. To determine the smallest set of independent physical modes, we should write a suitable expansion of fields and conjugate momenta and impose these constraint on them. As usual, the Fourier expansion is the suitable one, i.e.,

$$
\begin{aligned}
& A_{\mu}(x)=\frac{1}{(2 \pi)^{3 / 2}} \int d^{3} \tilde{k} e^{i \tilde{k} . \tilde{x}} a_{\mu}\left(\tilde{k}, x^{+}\right), \\
& \pi^{\mu}(x)=\frac{1}{(2 \pi)^{3 / 2}} \int d^{3} \tilde{k} e^{-i \tilde{k} . \tilde{x}} b^{\mu}\left(\tilde{k}, x^{+}\right) .
\end{aligned}
$$

Imposing the constraints and gauge fixing conditions on the physical modes $a_{\mu}$ and $b_{\mu}$, we find the following six conditions:

$$
\left\{\begin{array}{l}
b^{+}\left(\tilde{k}, x^{+}\right)=0, \\
b^{i}\left(\tilde{k}, x^{+}\right)=-i\left(k_{-} a_{i}\left(-\tilde{k}, x^{+}\right)-k_{i} a_{-}\left(-\tilde{k}, x^{+}\right)\right), \\
-i k_{-} b^{-}\left(\tilde{k}, x^{+}\right)=i k_{i} b^{i}\left(\tilde{k}, x^{+}\right), \\
a_{-}\left(\tilde{k}, x^{+}\right)=0, \\
b^{-}\left(\tilde{k}, x^{+}\right)=i k_{-} a_{+}\left(-\tilde{k}, x^{+}\right) .
\end{array}\right.
$$

There remain two independent physical modes which can be chosen as $a_{1}\left(\tilde{k}, x^{+}\right)$and $a_{2}\left(\tilde{k}, x^{+}\right)$. Here, noticing that the field elements are real functions, we construct a linear superposition of these independent modes in a conjugate way as

$$
\begin{gathered}
a\left(k, x^{+}\right)=a_{1}\left(k, x^{+}\right)+i a_{2}\left(k, x^{+}\right) \\
a^{\dagger}\left(k, x^{+}\right)=a_{1}\left(-k, x^{+}\right)-i a_{2}\left(-k, x^{+}\right) .
\end{gathered}
$$

Rewriting physical modes according to this set of independent modes, we have

$$
\begin{aligned}
a_{-}\left(k, x^{+}\right) & =b^{+}\left(k, x^{+}\right)=0 \\
a_{+}\left(k, x^{+}\right) & =-\left(\frac{k_{1}-i k_{2}}{2 k_{-}}\right) a\left(k, x^{+}\right)-\left(\frac{k_{1}+i k_{2}}{2 k_{-}}\right) a^{\dagger}\left(-k, x^{+}\right) \\
b^{1}\left(k, x^{+}\right) & =\frac{-i k_{-}}{2}\left(a\left(-k, x^{+}\right)+a^{\dagger}\left(k, x^{+}\right)\right) \\
b^{2}\left(k, x^{+}\right) & =\frac{-i k_{-}}{2}\left(a\left(-k, x^{+}\right)-a^{\dagger}\left(k, x^{+}\right)\right) \\
b^{-}\left(k, x^{+}\right) & =\frac{i}{2}\left(\left(k_{1}-i k_{2}\right) a\left(-k, x^{+}\right)\right. \\
& \left.+\left(k_{1}+i k_{2}\right) a^{\dagger}\left(-k, x^{+}\right)\right) .
\end{aligned}
$$

To this end, we can construct the symplectic two-form as

$$
\begin{aligned}
\Omega & =\int d^{3} \tilde{x} 2\left(d \pi^{\mu} \wedge d A_{\mu}\right) \\
& =\int d^{3} k\left(-i k_{-}\right)\left(d a^{\dagger}\left(k, x^{+}\right) \wedge d a\left(k, x^{+}\right)\right) .
\end{aligned}
$$

Using the inverse of the symplectic matrix (see the Appendix B), we find the Dirac brackets of physical modes as

$$
\left\{a\left(k, x^{+}\right), a^{\dagger}\left(k^{\prime}, x^{+}\right)\right\}=\frac{-1}{i k_{-}} \delta\left(k_{-}-k_{-}^{\prime}\right) \delta^{2}\left(k_{\perp}-k_{\perp}^{\prime}\right) .
$$

The canonical Hamiltonian in terms of the physical modes can be written as

$$
H_{c}=\int d^{3} k\left(\frac{k_{1}^{2}+k_{2}^{2}}{2}\right)\left(a^{\dagger}\left(k, x^{+}\right) a\left(k, x^{+}\right)\right) .
$$

In contrast to conventional coordinates [9], the Hamiltonian (84) is diagonal in terms of the transverse modes $a\left(\tilde{k}, x^{+}\right)$ and $a^{\dagger}\left(\tilde{k}, x^{+}\right)$. In other words, the transverse modes appear in light-cone coordinates in a natural way and we need not to choose any polarization direction to quantize the theory. In fact, by eliminating the redundant modes due to the lightcone constraints we need not to assume any polarization direction (as is done for instance in light-cone spinor field where the summation over spin indices has been eliminated).

Using the Hamiltonian of Eq. (84) and the Dirac brackets (83), the equations of motion of physical modes read 


$$
\begin{gathered}
\partial_{+} a\left(\tilde{k}, x^{+}\right)=\left\{a, H_{c}\right\}=i \omega_{+} a\left(\tilde{k}, x^{+}\right), \\
\partial_{+} a^{\dagger}\left(\tilde{k}, x^{+}\right)=\left\{a^{\dagger}, H_{c}\right\}=-i \omega_{+} a^{\dagger}\left(\tilde{k}, x^{+}\right),
\end{gathered}
$$

where $\omega_{+}=\frac{k_{1}^{2}+k_{2}^{2}}{2 k_{-}}$. Inserting the solutions of Eqs. (85) and (86) in the expansions (76) and (77) of fields, we find nonvanishing components of electromagnetic fields as

$$
\begin{aligned}
A_{+}(x)= & \frac{1}{(2 \pi)^{3 / 2}} \int d^{3} k(-1) e^{i k x} \\
\times & \left(\left(\frac{k_{1}+i k_{2}}{2 k_{-}}\right) a(k)-\left(\frac{k_{1}+i k_{2}}{2 k_{-}}\right) a^{\dagger}(-k)\right), \\
A_{1}(x) & =\frac{1}{(2 \pi)^{3 / 2}} \int d^{3} k e^{i k x} \frac{1}{2}\left(a(k)+a^{\dagger}(-k)\right), \\
A_{2}(x) & =\frac{1}{(2 \pi)^{3 / 2}} \int d^{3} k e^{i k x} \frac{-i}{2}\left(a(k)-a^{\dagger}(-k)\right),
\end{aligned}
$$

where $a(k) \equiv a_{i}(k, 0)$ are Schrödinger modes. For nonvanishing components of momentum fields, we have also the following expansions:

$$
\begin{aligned}
\pi^{-}= & \frac{1}{(2 \pi)^{3 / 2}} \int d^{3} k e^{-i k x} \frac{i}{2}\left(\left(k_{1}-i k_{2}\right) a(-k)\right. \\
& \left.+\left(k_{1}+i k_{2}\right) a^{\dagger}(-k)\right) \\
\pi^{1}= & \frac{1}{(2 \pi)^{3 / 2}} \int d^{3} k e^{-i k x} \frac{-i k_{-}}{2}\left(a(-k)+a^{\dagger}(k)\right) \\
\pi^{2}= & \frac{1}{(2 \pi)^{3 / 2}} \int d^{3} k e^{-i k x} \frac{-i k_{-}}{2}\left(a(-k)-a^{\dagger}(k)\right) .
\end{aligned}
$$

Using brackets (83), we are able to calculate the Dirac brackets of the fields and conjugate momenta which is in complete agreement with known results [12]. These relations can be seen in Appendix B.

\section{LIGHT-CONE QUANTIZATION OF YANG-MILLS THEORIES}

In this section, we try to quantize non-Abelian YangMills theories using a symplectic method of quantization in light-cone coordinates. We will show that we are not able to impose constraints on the Fourier expansion of dynamical fields, but this is not a light-cone quantization problem. We will try to embed the light-cone non-Abelian Yang-Mills theories using the BFFT method of quantization [15].

Yang-Mills theories describe the behavior of elementary gauge particles intermediating the physical interactions given by the Lagrangian density

$$
\mathcal{L}=-\frac{1}{4} F_{a}^{\mu \nu} F_{\mu \nu}^{a}
$$

where $F_{\mu \nu}^{a}=\partial_{\mu} A_{\nu}^{a}-\partial_{\nu} A_{\mu}^{a}+g f_{b c}^{a} A_{\mu}^{b} A_{\nu}^{c}$, in which $g$ is the coupling constant, and $f_{a b}^{c}$ are the structure constants of a Lie group (i.e., the gauge group). The index $a$ runs over $1,2, \ldots, N$ where $N$ is the number of generators of the gauge group.

In conventional coordinates, this theory includes $N$ first class primary constraints and $N$ secondary first class constraints. Taking into account $2 \mathrm{~N}$ gauge fixing conditions, there remain $8 N-4 N$ d.o.f. [9]. However, in lightcone coordinates according to Eq. (5), we expect to have $2 N$ additional second class constraints which is half number of dynamical d.o.f.

The Lagrangian density in the light-cone coordinate reads

$$
\mathcal{L}=\frac{1}{2} F_{+-}^{a} F_{+-}^{a}+F_{-i}^{a} F_{+i}^{a}-\frac{1}{4}\left(F_{i j}^{a}\right)^{2} .
$$

The conjugate momentums are similar to Eqs. (66)-(68) with the additional subscript $a$ on the momentum fields $\pi_{a}^{\mu}$ conjugate to the fields $A_{\mu}^{a}$. Again, $\phi_{a}^{0} \equiv \pi_{a}^{+} \simeq 0$ and $\phi_{a}^{i} \equiv$ $\pi_{a}^{i}-F_{-i}^{a} \simeq 0$ are primary constraints and the total Hamiltonian reads

$$
\begin{aligned}
H_{T}= & \int d^{3} \tilde{x}\left(\frac{1}{2}\left(\pi_{a}^{-}\right)^{2}+\pi_{a}^{-}\left(D_{-}\right)^{a b} A_{+}^{b}+\pi_{a}^{i}\left(D_{i}\right)^{a b} A_{+}^{b}\right. \\
& \left.+\frac{1}{4} F_{i j}^{a} F_{i j}^{a}+u^{d}(x) \pi_{d}^{+}+v_{i}^{e}(x)\left(\pi_{e}^{i}-F_{-i}^{e}\right)\right),
\end{aligned}
$$

where $u^{d}(x)$ and $v_{i}^{e}(x)$ are Lagrange multipliers and $\left(D_{\nu}\right)^{a b} \equiv \delta_{b}^{a} \partial_{\nu}-g f_{b c}^{a} A_{\nu}^{c}$ is the covariant derivative. Assuming the fundamental Poisson brackets as

$$
\left\{A_{\mu}^{a}\left(\tilde{x}, x^{+}\right), \pi_{b}^{\nu}\left(\tilde{y}, x^{+}\right)\right\}=\delta_{\mu}^{\nu} \delta_{b}^{a} \delta^{3}(x-y),
$$

the consistency condition $\partial_{+} \phi_{a}^{0} \approx 0$ gives a set of secondary first class constraints as

$$
\chi^{a} \equiv\left(D_{i}\right)^{a b} \pi_{b}^{i}+\left(D_{-}\right)^{a b} \pi_{b}^{-} \approx 0 .
$$

Consistency condition of this secondary constraints holds identically. Consistency of the constraints $\phi_{a}^{i}$ determines the Lagrange multipliers $v_{i}^{e}$ via the relations

$$
2\left(D_{-}\right)^{a b} v_{i}^{b}+\left(D_{j}\right)^{a b} F_{i j}^{b}-\left(D_{i}\right)^{a b} \pi_{a}^{-}=0 .
$$

To construct the reduced phase space of the system, we need $2 N$ gauge fixing conditions conjugate to our $2 N$ first class constraints $\phi_{a}^{0}$ and $\chi^{a}$. To choose required gauge fixing conditions, we simply generalize the electromagnetic gauge fixing conditions and choose $\omega_{1}^{a} \equiv A_{-}^{a} \approx 0$ conjugate to $\phi_{a}^{0}$. The consistency condition of $\omega_{1}^{a}$ gives another gauge fixing condition as

$$
\omega_{2}^{a} \equiv \pi_{a}^{-}+\partial_{-} A_{+}^{a} \approx 0 .
$$


Hence, there are altogether $6 \mathrm{~N}$ conditions on the fields and conjugate momenta as

$$
\left\{\begin{array}{l}
\phi_{a}^{0} \equiv \pi_{a}^{+} \approx 0 \\
\phi_{a}^{i} \equiv \pi_{a}^{i}-F_{-i}^{a} \approx 0 \quad i=1,2 \\
\chi^{a} \equiv\left(D_{i}\right)^{a b} \pi_{b}^{i}+\left(D_{-}\right)^{a b} \pi_{b}^{-} \approx 0 \\
\omega_{1}^{a} \equiv A_{-}^{a} \approx 0 \\
\omega_{2}^{a} \equiv \pi_{a}^{-}+\partial_{-} A_{+}^{a} \approx 0
\end{array}\right.
$$

\section{A. Symplectic method}

In the scalar theory and electromagnetic field, we simply choose Fourier expansions of fields to find the independent physical modes. But in the non-Abelian Yang-Mills theories, there are some nonlinear terms in constraints such as $\phi_{a}^{i}=\pi_{a}^{i}-F_{-i}^{a}=\pi_{a}^{i}-\partial_{\mu} A_{\nu}^{a}+\partial_{\nu} A_{\mu}^{a}-g f_{b c}^{a} A_{\mu}^{b} A_{\nu}^{c}=0$

Due to the existence of nonlinear terms such $g f_{b c}^{a} A_{\mu}^{b} A_{\nu}^{c}$ we are not able to impose this constraint on the Fourier expansion of fields to construct the reduced phase space. However, this problem is not the problem of light-cone quantization and is the fundamental problem of quantization of non-Abelian Yang-Mills theories. To quantize this theory, one can set the limit $g=0$ and quantize the theory perturbatively but attempt to quantize the theory directly fails due to lack of an appropriate expansion of fields which enables us impose the constraints.

In the next subsection, we try to embed non-Abelian Yang-Mills theories to an extended phase space to see the problem from another point of view.

\section{B. BFFT method}

In this subsection, we try to embed non-Abelian Yangmills theories in an extended phase space in which second class constraints, i.e., $\phi_{a}^{i}$ become first class constraints using BFFT method $[15,16]$. In this method, first of all, we need to extend phase space by adding some extra fields $(q, p) \oplus \eta$. the number of these auxiliary fields are equal to the number of second classs constraints appear in original phase space. By introducing $\omega_{\alpha \beta}$ as the algebra of new variables $\left\{\eta_{\alpha}, \eta_{\beta}\right\}=\omega_{\alpha \beta}$ and $\Delta_{\alpha \beta}=\left\{\tau_{\alpha}^{(0)}, \tau_{\beta}^{(0)}\right\}$ where $\tau_{\alpha}(q, p, \eta)$ is our constraint in embedded phase space and $\tau_{\alpha}^{(n)}$ are the $n$th order of expansion of embedded constraints according to new variables $\eta$, we have

$$
\begin{gathered}
\tau_{\alpha}^{(1)}=\chi_{\alpha}^{\beta}(q, p) \eta_{\beta}, \\
\Delta_{\alpha \beta}+\chi_{\alpha}^{\gamma} \omega_{\gamma \lambda} \chi_{\beta}^{\lambda}=0 .
\end{gathered}
$$

We are able to choose $\eta$ in such a way that the second class constraints become first class in new phase space. To find out these first class constraints, we have to solve the master equation, Eq. (103), according to $\chi_{\alpha}^{\gamma}$. We have

$$
\begin{gathered}
\Delta_{\alpha \beta}^{a b}=\left[\begin{array}{cc}
-\alpha^{a b} & 0 \\
0 & -\alpha^{a b}
\end{array}\right] \delta^{3}(x-y), \\
\alpha^{a b}=2\left(D_{-}\right)^{a b}=\left(2 \delta_{b}^{a} \partial_{-}-g f_{b c}^{a} A_{-}^{c}\right) .
\end{gathered}
$$

In order to solve Eq. (103), we need to guess $\omega_{\gamma \lambda}$. As shown in [15] by choosing $\omega_{\gamma \lambda}$ as below, the BFFT method become finite order. From now on, for simplicity, we drop out gauge indices $a$ and $b$.

$$
\omega_{\gamma \lambda}=\left[\begin{array}{cc}
1 & -1 \\
1 & 1
\end{array}\right],
$$

To solve (103), we consider $\chi_{\alpha}^{\gamma}$ as

$$
\chi_{\alpha}^{\gamma}=\left[\begin{array}{ll}
a_{1} & a_{2} \\
a_{3} & a_{4}
\end{array}\right] .
$$

By putting these relations in (103), we see that we have 4 unknown parameters $a_{i}$ and 3 equations, so we need to guess at least 1 of $a_{i}$ s to solve equations. Actually, different guess for these parameters and different solutions transform to each other by canonical transformations. We have

$$
a_{1}=0 \quad \& \quad a_{4}=0 \Rightarrow a_{3}=a_{2}=\sqrt{\alpha^{a b}} .
$$

So first class constraints in embedded phase space at first order can be written as

$$
\left\{\begin{array}{l}
\tau_{1}^{(1)}=\left(2 \delta_{b}^{a} \partial_{-}-g f_{b c}^{a} A_{-}^{c}\right)^{1 / 2} \eta_{2}^{b} . \\
\tau_{2}^{(1)}=\left(2 \delta_{b}^{a} \partial_{-}-g f_{b c}^{a} A_{-}^{c}\right)^{1 / 2} \eta_{1}^{b} .
\end{array}\right.
$$

By choosing $\omega_{\gamma \lambda}$ as Eq. (106), as shown in [15], the embedding become truncated and higher order in expansion $\tau_{\alpha}(q, p, \eta)=\sum_{n=0}^{\infty} \tau_{\alpha}^{(n)}$ vanishes. In relations (109), we see the square root of operator $\left(D_{-}\right)^{a b}$ which does not make sense well. To avoid this ambiguity, we are able to expand perturbatively with the assumption $\frac{g f_{b c}^{a} A c}{2 \delta_{b}^{a} \partial_{-}^{c}} \ll 1$ which is a true assumption in QCD for large momentums. Doing this and dropping out terms higher than $\frac{g f_{b c}^{a} c_{-}^{c}}{2 \delta_{b}^{c} \partial_{-}} \ll 1$, we have

$$
\left\{\begin{array}{l}
\tau_{1}^{(1)}=\left(\left(2 \delta_{b}^{a} \partial_{-}\right)^{1 / 2}-\left(\frac{1}{2} \frac{g f_{b c}^{a} A_{-}^{c}}{\left(2 \delta_{b}^{a} \partial_{-}\right)^{1 / 2}}\right)\right) \eta_{2}^{b} \\
\tau_{2}^{(1)}=\left(\left(2 \delta_{b}^{a} \partial_{-}\right)^{1 / 2}-\left(\frac{1}{2} \frac{g f_{b c}^{a} A_{-}^{c}}{\left(2 \delta_{b}^{a} \partial_{-}\right)^{1 / 2}}\right)\right) \eta_{1}^{b}
\end{array}\right.
$$

The above relations are embedded constraints in extended phase space which are first class. Note that we 
obtained these constraint perturbatively and these are not exact.

Comparing two methods discussed in this section, we see that in the case of symplectic method for quantizing nonAbelian Yang-Mills theories, due to nonlinear terms in constraints (100), there is not any appropriate expansion of fields which enables us to obtain the smallest set of physical modes unless we put $g=0$. But in the BFFT method, although we cannot find embedded constraint exactly, we can find them perturbatively. This is remarkable consequence.

\section{CONCLUSION}

The appearance of additional constraints on a field theory in light-cone coordinates is well known. However, for the expansions of fields and conjugate momenta in light-cone coordinates according to physical modes, authors use the usual expansions of conventional coordinates with an extra Heaviside step function. This step function divides the momentum space into two parts which enables us to choose say $k_{-}>0$ part of the momentum space. In this paper, we have proposed alternative expansions of the fields and conjugate momenta on the whole momentum space for the scalar, fermionic and vector fields. Our expansion is based on the fact that the number of independent physical modes must be equal to the number of d.o.f. in phase space. To do this, we exactly have investigated the dynamical structure of phase space variables by enumerating the number of d.o.f. as well as independent physical modes as follows.

First of all, we have shown that nondiagonal form of the light-cone metric causes changes in the constraint structure of the field theories described by the quadratic and firstorder Lagrangians. We showed exactly that half of the dynamical equations of motion are replaced by the lightcone constraints, hence the number of dynamical d.o.f. is divided by two, comparing with the conventional coordinates. Although this phenomenon is met by physicists working on concrete models [5], it is not clearly recognized as a general role for an arbitrary model. We showed that the light-cone constraints together with the remaining half of the dynamical equations of motion are equivalent to the whole equations of motion in conventional coordinates.

Second, since the number of independent physical modes are equal to the number of d.o.f., using the symplectic method of quantization, we chose the most appropriate set of independent physical modes to expand the phase space variables. By imposing the constraints on the phase space variables to obtain the reduced phase space, we priori have solved half of the equations of motion. By solving the remaining equations of motion, we obtained Schrödinger modes. Then we showed that each one of Schrödinger modes can play the role of creation or annihilation operator depending on the sign of the $k_{-}$ component of the momentum vector.

At the end, using symplectic method of quantization and analysing the dynamical structure of the phase space variables, we have proposed alternative expansions of the fields in the whole momentum space with true number of physical modes. Notice that the number of independent physical modes must be equal to the number of independent phase space variables. In the case of the scalar field, we have obtained relations (24) which shows that only one set of Schrödinger modes, i.e., $a(k)$ act as ladder operators. In the cases of fermionic and vector fields, our expansions have more significant remarks.

In the case of fermionic fields, we have obtained relations (64) for phase space variables. Additional lightcone constraints eliminate summation over spin indices in these expansions. We showed that ladder operators in the quantum theory create and annihilate particles and antiparticles in a specific superposition of spin indices.

Similar situation arises in the case of the vector field. As illustrated in the relations (87), (88), (89), the summation over polarization states is no longer necessary in the expansions of the fields and conjugate momenta.

We also investigated the constraint structure of the nonAbelian Yang-Mills theories in light-cone coordinates and showed that the number of d.o.f. is again half of those of conventional coordinates. Due to the existence of nonlinear terms such $g f_{b c}^{a} A_{\mu}^{b} A_{\nu}^{c}$ in the expressions of the constraints, we are not able to impose the constraints on the Fourier expansion of the fields to construct the reduced phase space. However, this problem is not due to light-cone quantization and is the fundamental problem of quantization of non-Abelian Yang-Mills theories. To quantize this theory, one can set the limit $g=0$ and quantize the theory perturbatively. However, any attempt to quantize the theory directly fails due to lack of appropriate expansions of the fields which enable us to impose the constraints.

Same problem arose in BFFT embedding of non-Abelian Yang-Mills Theories due to square root of operator $\left(2 \delta_{b}^{a} \partial_{-}-g f_{b c}^{a} A_{-}^{c}\right)^{1 / 2}$, so we are not able to quantize the theory exactly and nonperturbatively.

\section{ACKNOWLEDGMENT}

The authors thank $\mathrm{H}$. Ghaemi for valuable discussions.

\section{APPENDIX A: SYMPLECTIC METHOD OF QUANTIZATION}

In this Appendix, we briefly review the symplectic method of quantization which was proposed originally by Faddeev and Jackiw [17] and was shown to be equivalent to Dirac's method of quantization of constrained systems [18]. For a comprehensive review of this method, see Ref. [19]. 
For practical purposes, the symplectic method is based on the following steps:

(A) First, we should determine the complete constraint structure of systems [11]. This means that we should determine the primary constraints by calculating conjugate momenta. Then by applying the consistency conditions and defining fundamental Poisson brackets of canonical variables, we should obtain the secondary constraints. In this step, we also need to construct the canonical Hamiltonian of the system.

After investigating the constraint structure of the system, we should classify constraints as first and second class constraints using fundamental Poisson brackets. According to a conjecture by Dirac [11], first class constraints are the generators of gauge transformations. In this way, we should fix the gauges by imposing additional gauge fixing conditions on the system. In Ref. [20], the essential requirements for an appropriate gauge fixing conditions are given.

(B) In the second step, we should propose appropriate expansions of the fields and which enables us to impose constraints and gauge fixing conditions to get the reduced phase space. This step determines physical modes as the smallest set of time-dependent variables which uniquely describe every state of the classical system. In many familiar cases the Fourier expansion is an appropriate choice.

(C) This step is the most important part of the quantization procedure in which we find the canonical commutation relation of fields. The symplectic two-form is defined as [19]

$$
\Omega=\frac{1}{2} \int d^{3} x \sum_{i} d \pi^{i} \wedge d \phi_{i}
$$

Where $\phi_{i}$ and $\pi^{i}$ are fields and conjugate momentum fields respectively. By imposing constraints and gauge fixing conditions on the field expansions, we obtain them in terms of the physical modes. Using the expansions of the fields in terms of a set of physical modes, normally gives

$$
\Omega=\sum_{i, j} \int d^{3} k \omega_{i j} d a_{i}(k, t) \wedge d a_{j}(k, t)
$$

where $a_{i}$ are physical modes and $\omega_{i j}$ is the symplectic matrix. Finally by inverting the symplectic matrix, we get the Dirac brackets of the physical modes

$$
\left\{a_{i}(k, t), a_{j}\left(k^{\prime}, t\right)\right\}_{\mathrm{D} . \mathrm{B}}=\omega^{i j} \delta^{3}\left(k-k^{\prime}\right)
$$

where $\omega^{i j} \omega_{j k}=\delta_{k}^{i}$.

(D) In order to consider the dynamics of the theory, we should construct the canonical Hamiltonian of the system. Then we should write it in terms of physical modes derived in step (B). By solving the equations of motion of the physical modes based on the Dirac brackets in Eq. (A3), one can write the physical modes in terms of certain quantities at a given time, e.g., $a_{i}(k, 0)$. These quantities are called Schrödinger modes. The basic Algebra of Dirac brackets, i.e., Eq. (A3), can be written in terms of Schrödinger modes.

(E) Finally, using Dirac prescription of quantization, we quantize the theory according to

$$
\{,\} \rightarrow-i[,] .
$$

Note that by converting Schrödinger modes to operators, we will have the expansion of the fields in terms of creation and annihilation operators.

\section{APPENDIX B: DIRAC BRACKETS OF VECTOR FIELDS}

Using brackets (83), we are able to calculate the Dirac brackets of the fields as

$$
\begin{gathered}
\left\{A_{+}\left(\tilde{x}, x^{+}\right), \pi^{-}\left(\tilde{y}, x^{+}\right)\right\}=\frac{i}{2}\left|x^{-}-y^{-}\right| \theta\left(x^{-}-y^{-}\right) \partial_{\perp} \partial_{\perp} \delta^{2}\left(x^{\perp}-y^{\perp}\right) \\
\left\{A_{+}\left(\tilde{x}, x^{+}\right), \pi^{i}\left(\tilde{y}, x^{+}\right)\right\}=\frac{i}{2}\left|x^{-}-y^{-}\right| \theta\left(x^{-}-y^{-}\right) \partial_{i} \partial_{i} \delta^{2}\left(x^{\perp}-y^{\perp}\right) \\
\left\{A_{+}\left(\tilde{x}, x^{+}\right), A_{+}\left(\tilde{y}, x^{+}\right)\right\}=\frac{1}{2}\left|x^{-}-y^{-}\right|^{2} \theta\left(x^{-}-y^{-}\right) \partial_{\perp} \partial_{\perp} \delta^{2}\left(x^{\perp}-y^{\perp}\right) \\
\left\{A_{+}\left(\tilde{x}, x^{+}\right), A_{i}\left(\tilde{y}, x^{+}\right)\right\}=\frac{i}{2}\left|x^{-}-y^{-}\right| \theta\left(x^{-}-y^{-}\right) \partial_{i} \delta^{2}\left(x^{\perp}-y^{\perp}\right)
\end{gathered}
$$




$$
\begin{gathered}
\left\{A_{i}\left(\tilde{x}, x^{+}\right), A_{j}\left(\tilde{y}, x^{+}\right)\right\}=\frac{i}{2} \delta_{i}^{j} \theta\left(x^{-}-y^{-}\right) \delta^{2}\left(x^{\perp}-y^{\perp}\right) \\
\left\{A_{i}\left(\tilde{x}, x^{+}\right), \pi^{-}\left(\tilde{y}, x^{+}\right)\right\}=\frac{1}{2} \theta\left(x^{-}-y^{-}\right) \partial_{i} \delta^{2}\left(x^{\perp}-y^{\perp}\right) \\
\left\{A_{i}\left(\tilde{x}, x^{+}\right), \pi^{j}\left(\tilde{y}, x^{+}\right)\right\}=\frac{-1}{2} \delta_{i}^{j} \theta\left(x^{-}-y^{-}\right) \partial_{i} \delta^{2}\left(x^{\perp}-y^{\perp}\right) \\
\left\{\pi^{-}\left(\tilde{x}, x^{+}\right), \pi^{-}\left(\tilde{y}, x^{+}\right)\right\}=\frac{i}{2} \theta\left(x^{-}-y^{-}\right) \partial_{\perp} \partial_{\perp} \delta^{2}\left(x^{\perp}-y^{\perp}\right) \\
\left\{\pi^{-}\left(\tilde{x}, x^{+}\right), \pi^{i}\left(\tilde{y}, x^{+}\right)\right\}=\frac{-i}{2} \theta\left(x^{-}-y^{-}\right) \partial_{i} \partial_{i} \delta^{2}\left(x^{\perp}-y^{\perp}\right) \\
\left\{\pi^{i}\left(\tilde{x}, x^{+}\right), \pi^{j}\left(\tilde{y}, x^{+}\right)\right\}=\frac{i}{2} \delta_{i}^{j} \theta\left(x^{-}-y^{-}\right) \partial_{i} \partial_{i} \delta^{2}\left(x^{\perp}-y^{\perp}\right) .
\end{gathered}
$$

By transforming $\{,\} \rightarrow-i[$,$] according to the Dirac prescription of quantization, we finally achieve the quantized$ electromagnetic theory in light-cone coordinates.

[1] P. A. M. Dirac, Forms of relativistic dynamics, Rev. Mod. Phys. 21, 392 (1949).

[2] S. J. Brodsky, H. C. Pauli, and S. S. Pinsky, Quantum chromodynamics and other field theories on the light cone, Phys. Rep. 301, 299 (1998).

[3] M. B. Green and J. H. Schwarz, Covariant description of superstrings, Phys. Lett. 136B, 367 (1984).

[4] S. Alexandrov and S. Speziale, First order gravity on the light front, Phys. Rev. D 91, 064043 (2015).

[5] T. Heinzl, Light-cone quantization: Foundations and applications, in Methods of Quantization, Lecture Notes in Physics Vol. 572 (Springer, Berlin, Heidelberg, 2001), pp. 55-142, DOI: 10.1007/3-540-45114-5_2.

[6] S. Fubini and G. Furlan, Renormalization effects for partially conserved currents, Physics, Physique Fizika 1, 229 (1965).

[7] R. Dashen and M. Gell-Mann, Representation of Local Current Algebra at Infinite Momentum, Phys. Rev. Lett. 17, 340 (1966).

[8] J. B. Kogut and D. E. Soper, Quantum electrodynamics in the infinite-momentum frame, Phys. Rev. D 1, 2901 (1970).

[9] M. Henneaux and C. Teitelboim, Quantization of Gauge Systems (Princeton university, Princeton, NJ, 1992); K Sundermeyer, Lecture Notes in Physics, Constrained Dynamics (Springer-Verlag, Berlin, 1982), Vol. 169.

[10] M. Peskin and D. Schroeder, An introduction to quantum field theory (Avalon Publishing, New-York City, 1995).
[11] P. A. M. Dirac, Lectures on Quantum Mechanics (Yeshiva University Press, New York, 1964).

[12] P. P. Srivastava, Constraints and Hamiltonian in light-front quantized field theory, Il Nuovo Cimento A (1965-1970) 107, 549 (1994).

[13] M. Burkardt, Light front quantization, Advances in Nuclear Physics (Springer, Boston, MA, USA, 2002), pp. 1-74, DOI: 10.1007/0-306-47067-5_1.

[14] A. V. Popov, Symplectic approach to lightcone QCD, Nucl. Phys. A836, 136 (2010).

[15] M. Monemzadeh and A. Shirzad, Batalin-Fradkin-Tyutin method for mixed constrained systems and Chern-Simons theory, Phys. Rev. D 72, 045004 (2005).

[16] S. A. Nejad, M. Dehghani, and M. Monemzadeh, Lagrange multiplier and Wess-Zumino variable as extra dimensions in the torus universe, J. High Energy Phys. 18 (2018) 17.

[17] L. Faddeev and R. Jackiw, Hamiltonian Reduction of Unconstrained and Constrained Systems, Phys. Rev. Lett. 60, 1692 (1988).

[18] A. Shirzad and M. Mojiri, The difficulty of symplectic analysis with second class systems, J. Math. Phys. 46, 012702 (2005).

[19] A. Shirzad, A. Bakhshi, and Y. Koohsarian, Symplectic quantization of massive bosonic string in background B-field, Mod. Phys. Lett. A 27, 1250073 (2012).

[20] A. Shirzad, Full and partial gauge fixing, J. Math. Phys. 48, 082303 (2007). 